\title{
52 CELLULAR MECHANISMS OF MUSCLE INJURY DURING
}

\section{EXERCISE}

Michael Kalinski Professor of Exercise Physiology, School of Health Sciences, Kent State University, Kent, Ohio, USA

\subsection{6/bjsm.2010.078725.52}

We will describe the current understanding of the biochemical events following strenuous exercise, associated with overloading of the contractile elements and resulting in injury of skeletal muscle and inflammation. The extent of this damage depends on the mode, intensity, duration of exercise and prior exercise training. As a result of disruption of the muscle cell membrane, myocellular proteins pass through the membrane into the bloodstream. A variety of factors may potentially influence inflammatory responses to muscle damage after eccentric exercise: age, gender, anti-inflammatory drugs. Mild muscle damage stimulates muscle hypertrophy, whereas severe muscle damage can cause injury and may interfere with the training schedules of competitive athletes. 